\title{
Comparación de la dosis estimada en Recto y Vejiga con planificación de Braquiterapia 3D vs 2D
}

\section{Comparison of the estimated dose in the Rectum and Bladder with 3D versus 2D \\ brachytherapy planning}

*Correspondencia:

raulpuente.vallejo@gmail.com Teléfono [593] 0983301376

Conflicto de intereses: Los autores declaran no tener conflictos de intereses.

Fondos: Ver la página 42

Recibido: 19 Enero 2019

Aceptado: 11 Marzo 2019

Publicado: 30 Abril 2019

Membrete bibliográfico: Puente R, Nuñez C, Jaramillo C, Zurita K, Delos Reyes L, Défaz $Y$, Collantes V, García J, Pallaroso M, Molineris C. Comparación de la dosis estimada en Recto y Vejiga con planificación de Braquiterapia $3 \mathrm{D}$ vs 2D. Rev. Oncol. Ecu 2019;29(1):36-44.

ISSN: 2661-6653

DOI: https://doi.org/10.33821/301

Copyright Puente, et al. Este artículo es distribuido bajo los términos de Creative Commons Attribution License, el cual permite el uso y redistribución

\section{Raúl Puente Vallejo1*iD, Cristina Núñez Silva², Carolina Jaramillo Gómez² $^{2}$ Karina Zurita Vivero' ${ }^{1 D}$, Luis De Los Reyes Morales ${ }^{2}$, Yolanda Défaz Gómez², Vladimir Collantes Cruz² ${ }^{2}$, Jorge García Navas', Maira Pallaroso Yela3 ${ }^{3}$ Carlos Molineros Salgado3}

1. Universidad Central del Ecuador, Departamento de postgrados, Postgrado de radioterapia

2. Hospital Solón Espinosa Ayala, Solca núcleo de Quito, Servicio de Radioterapia.

3. Escuela Politécnica Nacional, Departamento de Postgrados, Maestría en física Médica.

\section{Resumen}

Introducción: En el tratamiento del Cáncer Cervico Uterino la braquiterapia (BQT) con planificación tridimensional (3D) está altamente extendida, aunque por su costo otros centros utilizan BQT con planificación bidimensional (2D). Es importante establecer si el beneficio que se obtiene con BQT en $3 \mathrm{D}$, en la protección a los órganos de riesgo, es un factor determinante para el cambio hacia esta nueva tecnología. El objetivo del estudio es comparar las dosis en ambos tipos de BQT en los órganos adyacentes al útero como el colon y vejiga.

Métodos: El presente estudio descriptivo, incluye pacientes con diagnóstico de cáncer de cuello uterino que recibieron BQT en el período comprendido de enero 2015 a diciembre 2018, en el servicio Radioterapia del Hospital Solón Espinosa Ayala, Quito-Ecuador. Las variables fueron la dosis recibida con planificaciones en 2D y 3D. Se obtuvo histogramas dosis volumen de los órganos de riesgo: recto y vejiga. Se comparan los promedios con T de Student.

Resultados: Participaron 44 casos, de $50.6 \pm 13.8$ años de edad. En estadío clínico IIB 24 casos ( $55 \%$ ). La dosis prescrita de BQT en el grupo fue de $683.6 \pm 27 \mathrm{cGY}$. El tipo de aplicador usado fue en la gran mayoría AyT de $5 \mathrm{~mm}$ en 34 casos (77\%). La mayoría de los tratamientos consistieron en terapias de 
4 sesiones 32 casos ( $73 \%$ ), con 117 implantes para BQT. La dosis planificada en vejiga en 2D fue 5.5 Gy, versus 5.1 Gy en 3D, $P=0.01$. La dosis planificada en recto en $2 \mathrm{D}$ fue $2.8 \mathrm{~Gy}$; versus 3.0 Gy en 3D $P=0.06$.

Conclusión: La planificación en 3D reduce significativamente 0.4 Gy la dosis irradiada a vejiga. En el caso del recto la dosis la dosis no produce cambios estadísticos respecto a planificación en 2D.

Palabras Claves: BRAQUITERAPIA, CUELLO DEL ÚTERO, NEOPLASIAS DEL CUELLO UTERINO, COLON, RADIOTERAPIA, PLANIFICACIÓN DE LA RADIOTERAPIA ASISTIDA POR COMPUTADOR

DOI: $10.33821 / 301$

\section{Abstract}

Introduction: In the treatment of Uterine Cervical Cancer brachytherapy (BQT) with three-dimensional planning (3D) is highly widespread, although for its cost other centers use BQT with two-dimensional planning (2D). It is important to establish if the benefit obtained with BQT in $3 \mathrm{D}$, in the protection of risk organs, is a determining factor for the change towards this new technology. The aim of the study is to compare the doses in both types of BQT in the organs adjacent to the uterus such as the colon and bladder.

Methods: The present descriptive study includes patients diagnosed with cervical cancer who received BQT in the period from January 2015 to December 2018, in the Radiotherapy service of the Hospital Solón Espinosa Ayala, Quito-Ecuador. The variables were the dose received with 2D and 3D plans. Histograms were obtained volume volume of the organs at risk: rectum and bladder. The averages are compared with Student's T.

Results: There were 44 cases, of $50.6 \pm 13.8$ years of age. In clinical stage IIB 24 cases (55\%). The prescribed dose of BQT in the group was $683.6 \pm 27 \mathrm{cGY}$. The type of applicator used was in the great majority AyT of $5 \mathrm{~mm}$ in 34 cases (77\%). The majority of treatments consisted of therapies of 4 sessions 32 cases (73\%), with 117 implants for BQT. The planned dose in 2D bladder was $5.5 \mathrm{~Gy}$, versus $5.1 \mathrm{~Gy}$ in $3 \mathrm{D}, \mathrm{P}=0.01$. The planned dose in rectum in $2 \mathrm{D}$ was $2.8 \mathrm{~Gy}$; versus $3.0 \mathrm{~Gy}$ in $3 \mathrm{D} \mathrm{P}=$ $0.06)$.

Conclusion: 3D planning significantly reduces $0.4 \mathrm{~Gy}$ the dose irradiated to the bladder. In the case of the rectum, the dose does not produce statistical changes with respect to $2 \mathrm{D}$ planning.

Keywords: BRACHYTHERAPY, CERVIX UTERI, UTERINE CERVICAL NEOPLASMS, COLON, RADIOTHERAPY, RADIOTHERAPY PLANNING COMPUTER-ASSISTED

DOI: $10.33821 / 301$

\section{Introducción}

El cáncer de cuello uterino en el Ecuador ocupa el segundo lugar en incidencia en tumores malignos en nuestra población femenina [1], el manejo de esta entidad requiere de un enfoque multidisciplinario, en los estadios localmente avanzados, el abordaje es fundamentalmente la radioterapia y quimioterapia radiosensibilizante [2]. 
La administración de radioterapia para el cáncer de cérvix localmente avanzado busca llegar a dosis por sobre $80 \mathrm{~Gy}$, lo que se logra asociando la administración de radioterapia externa y braquiterapia intracavitaria [3].

La braquiterapia intracavitaria ha presentado en la última década un importante cambio, incorporando los sistemas de planificación inversa, en tres dimensiones [4]; gracias a los cuales hoy en día se la puede realizar utilizando imágenes de tomografía o resonancia magnética para la definición de volúmenes y la determinación de histogramas dosis volumen en las regiones tumorales; así como en los órganos de riesgo.

A diferencia de lo que sucedía en los sistemas tradicionales de braquiterapia en dos dimensiones, donde el cálculo de la dosis y la estimación de la misma, en los órganos tanto tumorales como de riesgo, se basaba en puntos biplanares dados por el cruce de imágenes de rayos $X$ bidimensionales [3].

El histogramas dosis volumen permite conocer una estimación mucho más real de la cantidad de radiación que reciben los órganos de riesgo, con lo que se esperaría una mayor protección de los mismos [5].

Sin embargo la baja incidencia de complicaciones severas que han presentado las pacientes al igual que los reportes a nivel mundial con la aplicación de braquiterapia en dos dimensiones [6], nos llevó a cuestionarnos si la dosis que llega a los órganos de riesgo utilizando el sistema de planificación en dos dimensiones es significativamente mayor a la que se logra con la planificación tridimensional. Consideramos importante establecer si el beneficio que se obtiene con braquiterapia en tres dimensiones, en la protección a los órganos de riesgo, es un factor determinante para el cambio hacia esta nueva tecnología, ya que todavía en muchos países en vías de desarrollo sigue siendo el sistema convencional bidimensional la única forma en que se administra braquiterapia [7].

El objetivo del estudio es comparar las dosis en ambos tipos de braquiterapia en los órganos adyacentes al útero como el colon y vejiga.

\section{Materiales y Métodos}

El presente estudio observacional de cohorte transversal, se realizó en el servicio Radioterapia del Hospital Solón Espinosa Ayala, Solca núcleo de Quito, Ecuador. Ingresaron al estudio todos los registros de las pacientes con diagnóstico de Cáncer de cuello Uterino que fueron sometidas a Braquiterapia en el período comprendido desde enero del 2015 a diciembre del 2018.

El universo estuvo conformado de todos los pacientes que ingresaron al servicio con tumores de cuello uterino que tuvieron todos los datos analizables. El muestreo fue no probabilístico de tipo intencional. 
Las variables documentadas fueron la dosis recibida en las dos terapias. Por ser estudio descriptivo el estudio no requirió consentimiento informado y aprobación del comité de ética de la institución.

Los casos fueron tratados con braquiterapia, brevemente la planificación de tratamiento en tres dimensiones fue basada en tomografía computarizada [8]; de pacientes ya tratadas a planificación en dos dimensiones determinando los puntos $A, B$, punto recto y punto vejiga $[9,10]$, en cada uno de los implantes.

Una vez generado esta transformación virtual se volvió a trasladar esta planificación basada en puntos, al sistema en tres dimensiones y obtuvimos histogramas dosis volumen de los órganos de riesgo: recto y vejiga.

Generando así una estimación en 2cc de volumen tanto en recto y vejiga de la dosis que virtualmente estaría recibiendo la paciente si se hubiese realizado cada implante con planificación en dos dimensiones.

Se utiliza análisis descriptivo con frecuencias y porcentajes. Mediante el uso de la prueba estadística $\mathrm{T}$ de Student para diferencia de promedios de variables dependientes, se determinó la diferencia en el promedio de las dosis que recibió en 2 cc de recto y vejiga utilizando la planificación tridimensional y comparamos con los respectivos promedios que se obtuvieron de la transformación virtual de la planificación en dos dimensiones.

\section{Resultados}

Se incluyeron en el estudio 44 casos de mujeres con edad promedio de $50.6 \pm 13.8$ años. La mayoría de los casos estuvieron en estadío clínico IIB (Tabla 1). Las pacientes recibieron 117 implantes para Braquiterapia.

Tabla 1. Frecuencia de estadíos clínicos del grupo de estudio.

\begin{tabular}{|l|c|c|}
\hline Estadio & N & Porcentaje \\
\hline IIA & 1 & $2 \%$ \\
\hline IIB & 24 & $55 \%$ \\
\hline IIIB & 10 & $23 \%$ \\
\hline IS & 1 & $2 \%$ \\
\hline IVB & 5 & $11 \%$ \\
\hline IVA & 1 & $2 \%$ \\
\hline Sin datos & 2 & $5 \%$ \\
\hline
\end{tabular}

La dosis prescrita de braquiterapia en el grupo fue de $683.6 \pm 27$ cGY. El tipo de aplicador usado fue en la gran mayoría AyT de $5 \mathrm{~mm}$ en 34 casos (77 \%) (Tabla 2). La mayoría de los tratamientos consistieron en terapias de 4 sesiones 32 casos (73 \%) (Tabla 3). 
Tabla2. Tipo de aplicador usado en Braquiterapia.

\begin{tabular}{|l|c|c|}
\hline Tipo de aplicador & $\mathbf{N}$ & Porcentaje \\
\hline AyT $5 \mathrm{~mm}$ & 34 & $77 \%$ \\
\hline OyT $1.6 \mathrm{~cm}$ & 4 & $9 \%$ \\
\hline TyA $5 / 7 \mathrm{~mm}$ & 2 & $5 \%$ \\
\hline TyC $16 \mathrm{~mm}$ & 1 & $2 \%$ \\
\hline TyO $16 / 20 \mathrm{~mm}$ & 3 & $7 \%$ \\
\hline
\end{tabular}

Tabla 3. Número de sesiones de braquiterapia realizadas

\begin{tabular}{|l|c|c|}
\hline Número de BTR & Frecuencia & Porcentaje \\
\hline 1 implante & 2 & $5 \%$ \\
\hline 2 implantes & 4 & $9 \%$ \\
\hline 3 implantes & 5 & $11 \%$ \\
\hline 4 implantes & 32 & $73 \%$ \\
\hline 5 implantes & 1 & $2 \%$ \\
\hline
\end{tabular}

El promedio de dosis que recibieron recto y vejiga tanto en las planificaciones 3D y en la transformación virtual a planificaciones 2D, para cada uno de los órganos de riesgo se indica en la tabla 4.

La relación de estos promedios aplicando la $\mathrm{T}$ de Student para variables relacionadas, se muestra en la tabla 5.

Tabla 4. Promedio de dosis recibida por los órganos de riesgo para los diferentes planes y prescripciones.

\begin{tabular}{|l|c|c|c|}
\hline Órgano de riesgo & Plan 2D & Plan 3D & Dosis prescripta \\
\hline 2cc Vejiga & $5.5 \mathrm{~Gy}$ & $5.1 \mathrm{~Gy}$ & $7.0 \mathrm{~Gy}$ \\
\hline 2cc Recto & $2.8 \mathrm{~Gy}$ & $3.0 \mathrm{~Gy}$ & $7.0 \mathrm{~Gy}$ \\
\hline
\end{tabular}

Tabla 5. Comparación entre los promedios de dosis para los órganos de riesgo.

\begin{tabular}{|l|l|l|l|l|}
\hline Órgano de riesgo & Plan 2D & Plan 3D & IC 95\% & Valor P \\
\hline 2cc Vejiga 7.0 Gy & $5.5 \mathrm{~Gy}$ & $5.1 \mathrm{~Gy}$ & $(0.1 / 0.7)$ & 0.01 \\
\hline 2cc Recto 7.0 Gy & $2.8 \mathrm{~Gy}$ & $3.0 \mathrm{~Gy}$ & $(-0.4 / 0.009)$ & 0.06 \\
\hline
\end{tabular}

IC: Intervalo de confianza

\section{Discusión}

Con una prescripción de 7 Gy por implante, la planificación en 3 dimensiones reduce en promedio $0.4 \mathrm{~Gy}(P=0.01)$ a la dosis que recibe la vejiga por cada uno de estos, si tomamos 
en consideración que una paciente recibe en promedio 3 implantes, podremos extrapolar que este efecto reductor de dosis en la vejiga se multiplica.

En el caso del recto vemos un aumento en la dosis que recibe este, en la planificación en 3 dimensiones, ya que en promedio se aumenta $0.2 \mathrm{~Gy}(P=0.06)$ por implante, sin embargo en este caso no hay diferencia significativamente estadística.

Cabe señalar que esta es solamente una comparación dosimétrica generada virtualmente, ya que todas las pacientes fueron tratadas bajo planificación en 3 dimensiones. Se requerirá estudios que evalúen efectos adversos presentados en las dos modalidades de braquiterapia ginecológica, para poder determinar el impacto clínico de esta reducción de dosis en la vejiga observada dosimétricamente en nuestro estudio. Si bien este estudio nos da una aproximación dosimétrica del beneficio que se obtendría con la braquiterapia en dos dimensiones, en cuanto a reducción de dosis en órganos de riesgo, sería importante correlacionar estos datos con estudios que evalúen el beneficio dosimétrico en cuanto a cobertura de volumen tumoral.

A pesar de las mejoras dosimétricas que nos presenta la braquiterapia en tres dimensiones, la modalidad convencional sigue plenamente vigente en países en los que solo existe esta y se debe seguir utilizando, ya que el beneficio clínico que se obtiene con la braquiterapia en dos dimensiones está justificado [11].

\section{Conclusiones}

Con una prescripción de 7 Gy por implante, la planificación en 3 dimensiones reduce en promedio $0.4 \mathrm{~Gy}$ a la dosis que recibe la vejiga por tratamiento con diferencia estadística significativa. En el caso del recto la dosis la dosis aumenta 0.2 Gy por implante, sin diferencia estadística significativa.

\section{Agradecimientos}

Reconocemos a las personas que participaron indirectamente en el estudio tales como el personal técnico, pacientes y personal del Hospital Solón Espinosa Ayala, Solca núcleo de Quito, Servicio de Radioterapia.

\section{Información adicional}

\section{Abreviaturas}

BQT: Braquiterapia

IC: Intervalo de Confianza. 
Nota del Editor La Revista Oncología Ecu permanece neutral con respecto a los reclamos jurisdiccionales en mapas publicados y afiliaciones institucionales.

\section{Archivos Adicionales}

Ninguno declarado por los autores.

Fondos

Los fondos de la investigación fueron propios de los autores del presente artículo.

\section{Disponibilidad de datos y materiales}

Existe la disponibilidad de datos bajo solicitud al autor de correspondencia. No se reportan otros materiales.

\section{Contribuciones de los autores}

RPV, CNS, CJG, KZV realizaron la idea de investigación, revisión bibliográfica. LDLRM, YDG, VCC, JGN, MPY, CMS recolección de datos, escritura del artículo. RPV realizó el análisis crítico del artículo. CNS realizó las correcciones editoriales. Todos los autores leyeron y aprobaron la versión final del artículo.

Aprobación de ética y consentimiento para participar

No aplica a este estudio.

\section{Consentimiento para publicación}

No aplica.

\section{Información de los autores}

Raúl Puente Vallejo, Médico postgradista del tercer año de radioterapia Universidad Central del Ecuador- Solca, Núcleo de Quito, coordinador de la investigación.

(D) https://orcid.org/0000-0002-0678-2571

Cristina Núñez Silva, Médico especialista en radioterapia tratante Hospital Solón Espinosa Ayala, Solca, núcleo de Quito.

Carolina Jaramillo Gómez, Médico especialista en radioterapia tratante Hospital Solón Espinosa Ayala, SOLCA núcleo de Quito. 
Karina Zurita Vivero, Médico postgradista del tercer año de radioterapia Universidad Central del Ecuador- SOLCA Núcleo de Quito.

Luis De Los Reyes Morales, Médico especialista en radioterapia tratante y jefe de servicio Hospital Solón Espinosa Ayala, SOLCA núcleo de Quito.

Yolanda Défaz Gómez, Física master en física médica Hospital Solón Espinosa Ayala, SOLCA núcleo de Quito.

Vladimir Collantes Cruz, Ingeniero químico master en física médica Hospital Solón Espinosa Ayala, SOLCA núcleo de Quito.

Jorge García Navas, Físico, cursante de la maestría en física medica Escuela Politécnica Nacional- SOLCA Núcleo de Quito.

Maira Pallaroso Yela, Física, cursante de la maestría en física medica Escuela Politécnica Nacional- SOLCA Núcleo de Quito.

Carlos Molineros Salgado, Físico, cursante de la maestría en física medica Escuela Politécnica Nacional- SOLCA Núcleo de Quito.

Revisiones por pares

Acceda a la revisión de pares académicos en el siguiente enlace: https://publons.com/review/4519391/

\section{Referencias}

Abreviaturas en la referencias DOI: Digital Object Identifier PMID: PubMed Identifier SU: Short URL
1. Cueva P, Yépez J. Tumores. Sociedad de Lucha contra el Cáncer. Registro Nacional De Tumores / National Cancer Registry. 15th ed. Quito; 2014:118-125.

2. Chuag L, Temin S, Camacho R, Dueñas-Gonzales A, Feldman S, Gultekin M, et al. Management and Care of Women With Invasive Cervical Cancer: American Society of Clinical Oncology Resource-Stratified Clinical Practice Guideline. J Glob Oncol. 2014;118-25. DOI: 10.1200/JG0.2016.003954

3. European Society for Radiotherapy an Oncology. Report 89. Journal of the International Commission on Radiation Units and Measurements 2013;13(2):1-8. DOI: 10.1093/jicru/ndw042

4. Haie-Meder C, Pötter R, Van Limbergen E, Briot E, De Brabandere M, Dimopoulos J, et al. Recommendations from Gynaecological (GYN) GEC-ESTRO Working Group (I): Concepts and terms in 3D image based 3D treatment planning in cervix cancer brachytherapy with emphasis on MRI assessment of GTV and CTV. Radiother Oncol. 2005;74(3):235-45. DOI: 10.1016/j.radonc.2004.12.015

5. Harkenrider MM, Alite F, Silva SR, Small W. Image-based brachytherapy for the treatment of cervical cancer. Int J Radiat Oncol Biol Phys 2015;92(4):921-34. DOI: 10.1016/j.jijrobp.2015.03.010

6. Viswanathan AN, Lee LJ, Eswara JR, Horowitz NS, Konstantinopoulos PA, Mirabeau-Beale KL, et al. Complications of pelvic radiation in patients treated for gynecologic malignancies. Cancer. 2014;120(24):3870-83. DOI: $10.1002 /$ cncr.28849

7. Grover S, Xu MJ, Yeager A, Rosman L, Groen RS, Chackungal S, et al. A systematic review of radiotherapy capacity in low- and middle-income countries. Front Oncol. 2015;4:1-11. DOI: 10.3389/fonc.2014.00380 
8. Zolciak-Siwinska A, Gruszczynska E, Bijok M, Jonska-Gmyrek J, Dabkowski M, Staniaszek J, et al. Computed Tomography-Planned High-Dose-Rate Brachytherapy for Treating Uterine Cervical Cancer. Int J Radiat Oncol Biol Phys 2016;4:1-11. DOI: 10.1016/j.ijrobp.2016.04.025

9. Viswanathan AN, Thomadsen B. American Brachytherapy Society consensus guidelines for locally advanced carcinoma of the cervix. Part I: General principles. Brachytherapy. 2012;11:33-46. DOI: 10.1016/j.brachy.2011.07.003

10. Viswanathan AN, Beriwal S, De Los Santos J, Demanes J, Gaffney D, Hansen J, et al. American Brachytherapy Society consensus guidelines for locally advanced carcinoma of the cervix. Part II: Highdose-rate brachytherapy. Brachytherapy 2012;11:45-52. DOI: 10.1016/j.brachy.2011.07.002

11. Small W, Beriwal S, Demanes J, Dunsenbery K, Eifel P, Erickson B, et al. American Brachytherapy Society consensus guidelines for adjuvant vaginal cuff brachytherapy after hysterectomy. Brachytherapy 2012;11:58-67. DOI: 10.1016/j.brachy.2011.08.005. 